\title{
Xiaoping Dong, China CDC's Chief Expert of Virology
}

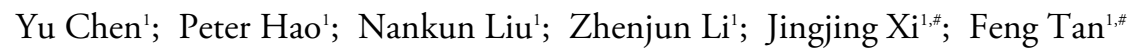



Xiaoping Dong is the Chief Expert of Virology of China CDC, Adjunct Professor at Beijing University and Xi'an Jiaotong University, and Adjunct Principal Investigator (PI) at the Chinese Academy of Science and China Academy of Chinese Medical Sciences. He is a syndic of the Chinese Society of Microbiology and the Head of the Group of Medical Virology. During his roughly 40-year career, he has dedicated himself to the studies of medical virology and molecular biology, particularly in the fields of prions, human papillomavirus (HPV), and many emerging infectious diseases. He has also participated in emerging responses and controls of many viral infectious diseases domestically and internationally.

In the period of 1980's and 1990's, his major research interest focused on human papillomavirus (HPV). With the help of DNA hybridization, Dong and his colleagues identified high positive rate of HPV DNAs in the tissues of cervical cancer. During 1991 to 1997, he went to Germany and explored the HPV carcinogenesis under the supervision of Prof. H. Pfister. Dong and the colleagues successfully mapped the binding sites of YY1, a new host factor, in viral long control region (LCR) and confirmed it as a negative regulator for the activity of HPV early promoter. This finding and several others supplied valuable evidence for carcinogenesis of HPV.

Under the guidance of Prof. T Hung, a famous senior Chinese virologist, Dong shifted his research to prion diseases $(\operatorname{PrD})$, when he returned from Germany in 1998. At that time, $\operatorname{PrD}$ was not widely recognized in China, and there was almost no capacity to diagnose human PrDs. Dong and his team started a mission to address this challenge by establishing various laboratory diagnostic tools. Chinese National Surveillance for Creutzfeldt-Jacob disease (CNS-CJD) was set up a couple of years later under the leadership of China CDC. With the innovative surveillance methodologies and working mechanism, as well as dozens of training programs, the annual number of suspected CJD cases increased from a small dozen in the beginning to more than 500 cases currently. More than 2,000 cases were diagnosed as sporadic CJD (sCJD) and about 250 cases were diagnosed as genetic $\operatorname{PrD}(\mathrm{gPrD})$. Such groundbreaking work supplies not only the platform for diagnosis of human PrDs, but also contributed to the recognition of China as a bovine spongiform encephalopathy (BSE)-free country. The accumulated data over the past roughly 20 years of CJD surveillance have helped explain the features of PrDs in China for the first time systematically and precisely.

Dong's team identified 19 different gPrD-associated mutations in Chinese people for the first time. The top 5 most frequent gPrDs in Chinese were T188K-gCJD (29.8\%), D178N-FFI (25.7\%), E200K-gCJD (18.8\%), E196A-gCJD (7.3\%) and P102L-GSS (6.4\%). Such pattern of PRNP mutants in Chinese gPrDs was not only completely distinct from that of Caucasian, but also different from Japanese and Korean. Some types of gPrDs showed geographic relationship, e.g., more D178N-FFI patients came from Henan and Guangdong, and more T188K-gCJD cases lived in the northern provinces. These findings and others of Chinese gPrDs enriched the knowledge of gPrDs worldwide.

After years of efforts, Dong and his team successfully set up two in vitro prion replication techniques, protein misfolding cyclic amplification (PMCA), and real-time quaking-induced conversion (RT-QuIC), which are already widely used in various studies. RT-QuIC has been used in the diagnosis for human PrDs, with specimens of the brain, cerebrospinal fluid (CSF), and skin. Additionally, a series of infectious cell models, animal models, and transgenic mice models infected with different prion strains had been established. Those techniques form the solid and unique research platform for prions in the levels of molecules, cells, tissues, and animals in vitro and in vivo.

In addition to human CJD surveillance, Dong and his team have several achievements in prion-related research including the following: 1) identification of autophagy activation in the brains of human PrDs and prion-infected rodent models; 2) global omics profiles that identified differentially expressed proteins and affected biological pathways; 3) aberrant alterations of biological functions in brains; 4) activation of brain native immunity; 
5) abnormal changes of protein post-translation modifications; and 6) cross-species transmission demonstrated by PMCA assisting prion strains to overcome species barriers. These accomplishments helped enrich the knowledge of prion biology and pathology.

In the past 20 years, Dong participated actively in the emerging response and control for many infectious diseases. In 2003, he dedicated himself to the fight against SARS. He went many times to the front lines in Guangdong, Beijing, and Shanxi for case investigation, epidemiological survey, and laboratory diagnosis. Working together with Sinovac and the Chinese Academy of Medical Science, he contributed greatly to the research and development (R\&D) of an inactivated SARS vaccine that was the first SARS vaccine to finish Phase I clinical trials worldwide. In an outbreak of human cases infected with H5N1 influenza virus in 2005, as the team leader from China side, he worked closely with the experts of the World Health Organization (WHO) for the investigations of the first two cases. He worked as a co-PI in the R\&D of an inactivated vaccine of $\mathrm{H} 5 \mathrm{~N} 1$ virus for human use, which successfully finished Phase I and II clinical trials. In 2015, he went to Sierra Leone as China CDC's team leader for Ebola disease. He and his colleagues set up a biosafety laboratory level III (BSL-3) in Freetown and conducted thousands of sample testing. Since the emergence of coronavirus disease 2019 (COVID-19) in 2020, Dong has fully dedicated himself to the national and international responses. He attended numerous domestic and international consultant Webinars, went the front lines in Beijing, Heilongiiang, Shaanxi, etc., contributed to the biweekly reports of the global COVID-19 pandemic, consulted for the R\&D of COVID-19 vaccines, and had several other contributions.

Dong has rich experience in international cooperation, which has led to his appointment as Director of Center for Global Public Health of China CDC since 2018. He and his colleagues visited many countries in Africa and Asia, communicated with different international organizations, drafted China CDC's global health strategic and development plan, created training programs, dispatched short- and long-term Chinese experts to different countries, and implemented international collaborating projects. New communication platforms have been developed and gradually recognized internationally, such as annual Consultation on Public Health Development (CPHD), roundtable of Belt \& Road Public Health Cooperation Network, program of Molecular Diagnosis and Pathogen Determination (ModPad), etc. The collaborating fields cover major infectious diseases, emerging responses, public health events, training and education, task-force capacity building, laboratory capacity, and others.

Dong has rich experience in medical virology and public health based on his long-term work in clinical medicine, laboratory, disease surveillance, and field emerging response. He has contributed significantly to academic research as a PI for more than 30 national and international projects and published more than 220 SCI-cited papers. Dong's international collaborative experience has provided him with wider perspective on medical virology and public health, and he will continue to use this expertise as China CDC's Chief Expert of Virology.

doi: $10.46234 / \mathrm{ccdcw} 2021.107$

\# Corresponding authors: Jingjing Xi, xijj@chinacdc.cn; Feng Tan, tanfeng@chinacdc.cn.

${ }^{1}$ Chinese Center for Disease Control and Prevention, Beijing, China.

Submitted: April 30, 2021; Accepted: May 03, 2021 